\title{
Towards Applying CSCW in Improving Orthography System Development Process
}

\author{
${ }^{1}$ Sook Kuan Chin, ${ }^{2}$ Alvin W.Yeo, ${ }^{3}$ Nadianatra Musa \\ ${ }^{1,3}$ Faculty of Computer Science and Information Technology, \\ ${ }^{2}$ Institute of Social Informatics and Technological Innovations, \\ Universiti Malaysia Sarawak, \\ Sarawak, Malaysia \\ E-mail: ${ }^{1}$ s.kuan86@gmail.com, ${ }^{2}$ alvin@isiti.unimas.my, ${ }^{3}$ nadia@fit.unimas.my
}

\begin{abstract}
Orthography system development process is vital to help in the language documentation. Language documentation is useful to preserve the indigenous languages. There are many approaches that had been taken by the linguists to develop the orthography system. Unfortunately, the method that is used by the linguists to develop the orthography system is not feasible. As the linguists need to travel to the specific areas to collect and identify requirements of the languages, the whole process would be cumbersome. Thus, Computer Supported Cooperative Work (CSCW) had been chosen to apply in the orthography system development process. The purpose of applying CSCW into the orthography system development process is to use Information and Communication Technology (ICTs) to engage the linguists working together with the indigenous community.
\end{abstract}

Keywords: Orthography system development, CSCW, ICTs.

\section{INTRODUCTION}

Orthography system is known as the spelling system that consists of a set of symbols and writing rules to combine them to write words or sounds [1]. Orthography system provides the written form of the language so that native speakers able to document the indigenous languages. Since there are many indigenous languages still not yet documented, thus orthography system becoming increasingly important. However, orthography system development largely difficult as much time and money required for the linguists travel to rural remote area.

The focus of this paper is mainly on improving the performance of orthography system development. Orthography system development is a cumbersome process, as more time is required for linguists travel to rural remote area. Sometimes, linguists may spend few months or years to conduct the orthography system development process. Therefore, much costs spent in orthography system development as well.

Moreover, community participation is another critical factor in orthography system development. This is due to some of the indigenous communities are very passive in documenting their languages [2]. If they do not cooperate in orthography system development, it will delay the whole process. Besides that, the target languages tend to be spoken by people in remote rural areas with next to no Internet connectivity. These issues have degrades the performance of orthography system development. Thus, some improvements are needed to increase the possibility for orthography system development to be conducted at different places. So, ICTs was used to provide the online tool to enable collaborative working between linguists and community [3].

The location of this ongoing research is Sarawak, East Malaysia. The reason of choosing this location is because there are many indigenous minority languages in Sarawak do not as yet have agreed orthography system, as they have not traditionally been written down. Therefore, we choose to collaborate with the Penan community, a small minority indigenous group at Long Lamai, Sarawak.

Currently, there are many methods have been designed for orthography system development. However, they may not suitable if linguists are far apart from the community. Therefore, the new approach is proposed to improve the performance of orthography system development.

In this paper, Section II briefly discussed the related work of orthography system development. Section III shows the overview of Computer Supported Cooperative Work (CSCW). Section IV proposes a new way of improving orthography system development process and concludes this paper in Section VI.

\section{RELATED WORKS}

Several orthography system development processes have been introduced in this section. This is used to develop a clear common understanding so that we can implement the similar steps in proposed new approach. Thus, two minority languages were chosen, Temiar language and Bantu subgroup of NigerCongo languages.

\section{A. Temiar language}

Temiar language is a minority Austronesian language spoken by an "Orang Asli" (indigenous people) community in the Malay Peninsula (West Malaysia) [4]. Benjamin had developed a set of International Phonetic Alphabet (IPA) for this language. IPA covers all possible sounds of all human languages, and that only subset of these will be found within the phoneme inventory of particular languages [5]. On the

Exploratory Research Grant Scheme (ERGS) 